\title{
Research on the Intelligent Generation Method of 3D Virtual Vehicle
}

\author{
$\mathrm{Na} \mathrm{Guo*}$ \\ School of Mechanical, Electrical \& Information Engineering \\ Shandong University, Weihai \\ Guoxiaoyu2010@sina.com
}

Keywords: 3D animation, key frame, vector, dot product, cross product.

\begin{abstract}
In order to solve the problem that manual ways for the key frame of the vehicle moving animation needs to consume a lot of manpower and material resources during the 3D animation production. The key problem in this research is the control of the height of the vehicle and the rotation direction of the wheel. The height of a vehicle can be transformed to the distance between he wheel center to center point of Intersection line of the wheel and ground.The wheel rotation direction is obtained by the vector dot product and cross product. The research result can make the vehicle automatically forward,backward,uphill,downhill with high efficiency and good quality.
\end{abstract}

\section{Introduction}

In the three-dimensional video animation creation, vehicle animation production often need professional animators take a very long time to manually make key frames in the 3D software , especially complex road conditions, such as downhill and uphill, mountain areas, or the number of vehicles more. Vehicle animation production often spends a lot of manpower and material resources, and the quality is unsatisfactory frequently. In view of this problem, some research achievements have been made at home and abroad, but most of them are simple and can not meet the requirements of various driving conditions.

In this paper, a method of automatically generating vehicle moving animation in 3D virtual space is presented.It realizes the intelligent driving of the vehicle model in the $3 \mathrm{D}$ virtual scene, including the vehicle (wheel and vehicle body) forward, backward, uphill, downhill and so on. The wheel is always close to the ground, do not fall into the lower height, not empty. Body is always in the right position and angle. A virtual 3D vehicle travel intelligent controller was developed, the tool eliminates the need for a large number of manual key frame work, improve work efficiency and product accuracy.

\section{Algorithm design}

Suppose we know the starting point and the end point of the vehicle, as well as the time to travel between two points. The algorithm mainly solves two key problems, the first is the vehicle height control, the second is the wheel rotation angle and direction. Then, the algorithm is analyzed from the two aspects mentioned above.

Wheel height control.

Solution thought: control the height of the wheel, and then control the height of the whole vehicle.

On the ground $(\mathrm{Y}=0)$, it is as shown in Figure 1.

$$
\mathrm{H}=\mathrm{r}
$$

Notes: $\mathrm{H}$ is wheel center point height and $\mathrm{r}$ is wheel radius. 


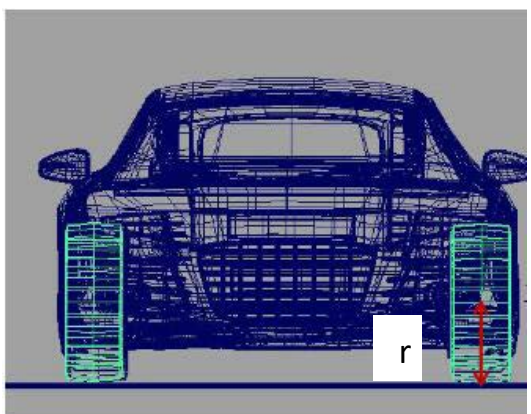

Fig. 1

(2)when the vehicle on the ground that is located in the high or low, the $\mathrm{Y}$ value is not equal to zero,as shown in Figure 2.

$$
\mathrm{H}=\mathrm{r}+\mathrm{h}
$$

Notes: $\mathrm{H}$ is wheel center point height, $\mathrm{r}$ is wheel radius and $\mathrm{h}$ is ground height.

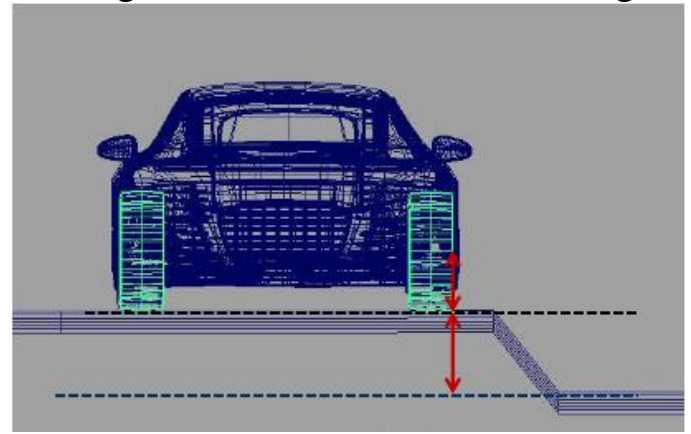

Fig. 2

(3)in complex traffic, such as viaduct or one side of higher ground ,vehicle roll, as shown in Figure 3, earlier formulas are not suitable.

Solution: $\mathrm{L}=\mathrm{r}$

$\mathrm{L}$ represents the diatance from the wheel center to the center point of the wheel and the ground line, instead of the wheel center height; $\mathrm{R}$ represents the wheel radius.

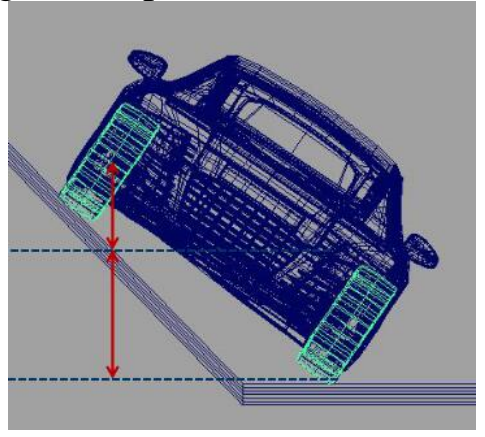

Fig. 3

In order to solve the problem conveniently, we create a rectangular patch Q.The patch center and the center of the wheel is a coincidence.And the two parallel edges of the $\mathrm{Q}$ is always perpendicular to the bottom of the car oAssuming that $C$ is the midpoint of the intersection line between the $\mathrm{Q}$ and the ground, the distance between the center of the wheel and the $\mathrm{C}$ is L.Regardless of the road conditions, the use of formula $\mathrm{L}=\mathrm{r}$, can control the height of the wheel, and then control the entire vehicle height. 


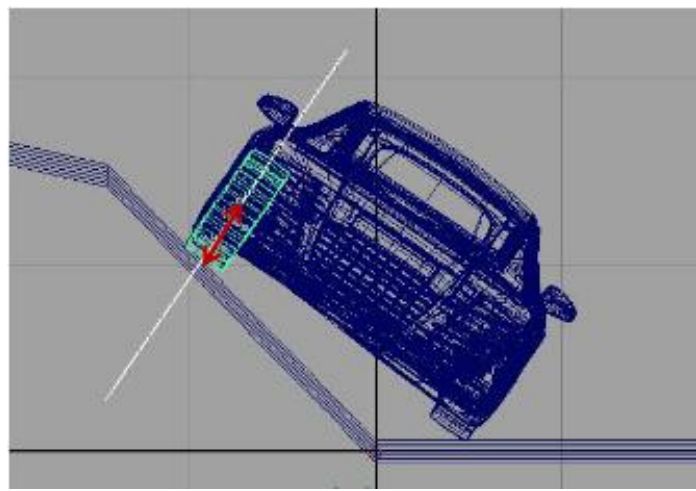

Fig. 4

\section{Rotation angle and the direction of the wheel.}

Place a square patch in the interior of the vehicle, and below red line indicates the side effects of the patch, The patch is perpendicular to the axis of the vehicle.

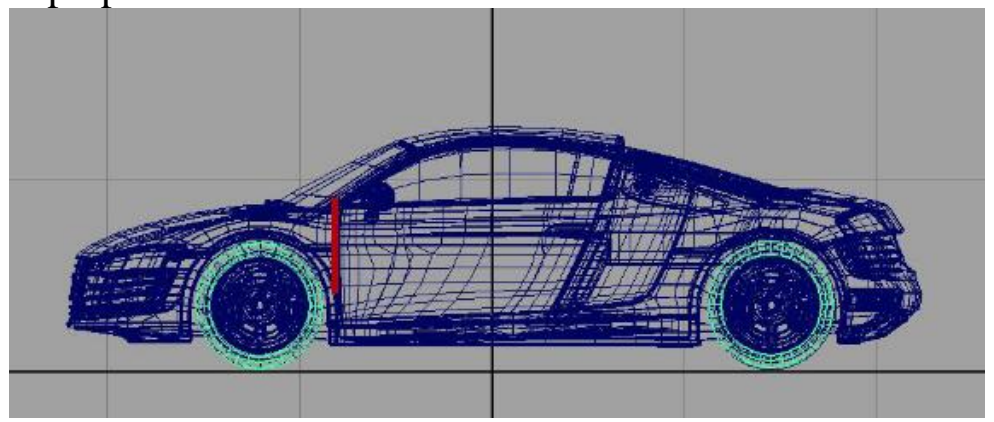

Fig. 5

The square patch is view as shown below, will do product vector A and vector B which are vector $\mathrm{C}$.

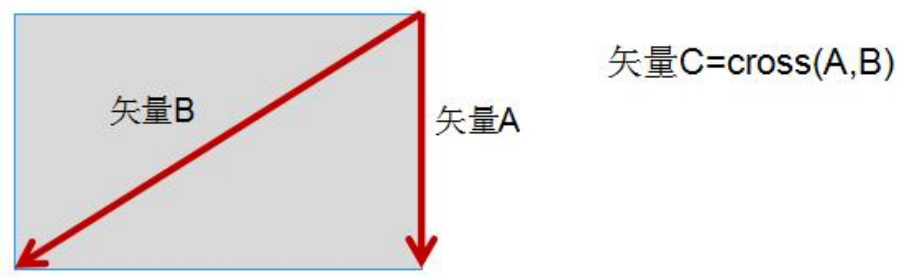

Fig. 6

The vehicle current position and the next frame position form a vector $\mathrm{D}$, as shown in Figure 7 , the vector $C$ and $D$ point multiplication, $E=\operatorname{dot}(C, d)$.

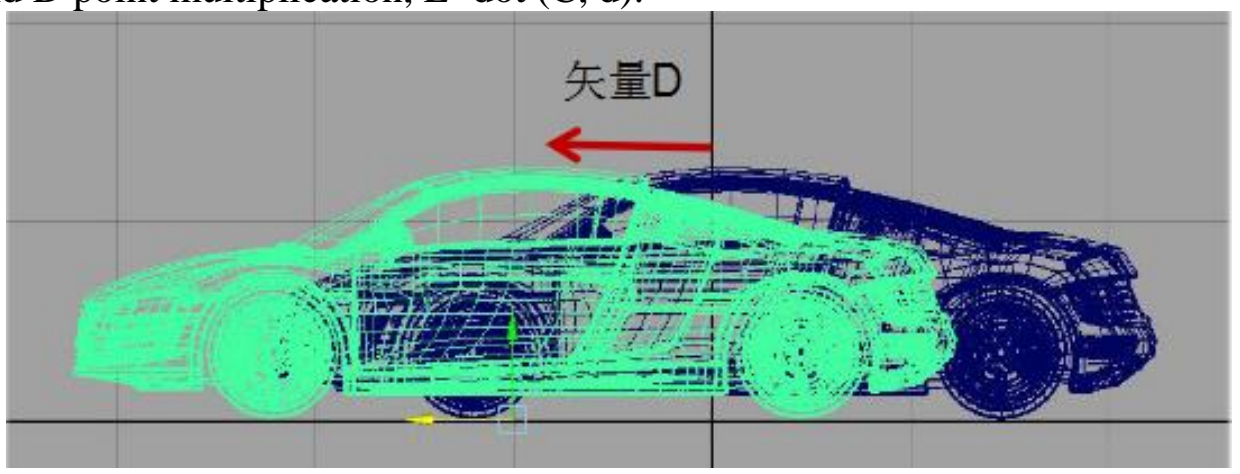

Fig. 7

Assuming the vehicle stay at the point $(\mathrm{x} 1, \mathrm{Y} 1, \mathrm{z} 1)$ at the next frame, at the current frame the vehicle position is $(\mathrm{x} 0, \mathrm{Y} 0, \mathrm{Z} 0)$, then distance between the two is $\mathrm{L}=$ sqrt (pow $(\mathrm{x} 1-\mathrm{x} 0,2)+$ pow $(\mathrm{z} 1-\mathrm{z} 0,2))$.

If $\mathrm{E}>0$, the angle from Vector $\mathrm{C}$ to vector $\mathrm{D}$ is more than 90 degrees, the vehicle should be move forward.During the current frame to the next frame, the wheel rotation angle is $L^{*} 180 /\left(r^{*} 3.14\right)$. 
If $\mathrm{E}<0$, the angle from Vector $\mathrm{C}$ to vector $\mathrm{D}$ is less than 90 degrees, the vehicle should be back.During During the current frame to the next frame , the wheel rotation angle is - $\mathrm{L}^{*} 180$ / $(\mathrm{r} * 3.14)$.

According to the above ideas, I have combined with the three-dimensional animation software environment, and developed a three-dimensional virtual vehicle driving intelligent control plug-in unit. Its main interface is shown in the following figure.

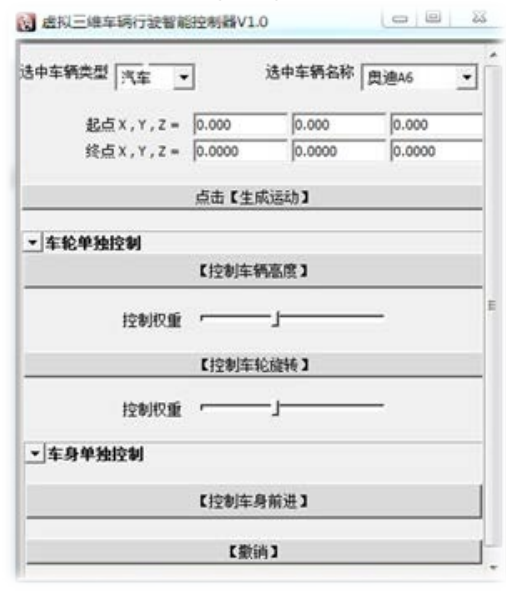

Fig. 8

Practice has proved that in a complex traffic scene with 10 racing,The animators will spend 21 hours for a period of 10 seconds of animation。But using the plugin, you can complete the task within 10 minutes.

\section{Conclusions}

In this paper, for the vehicle 3D animation production manual method is time-consuming and laborious and the quality is not high, the $3 \mathrm{D}$ virtual intelligent vehicle driving method is proposed, including vehicle height control algorithm and the wheel driving direction and angle of the algorithm. For the vehicle height, according to the general idea it can be obtained by solving the height of wheel center point. But it has a great influence on the land condition, especially the slope, the high ground, the depression and so on.However, the distance from the center of a wheel to center point of the intersection line between the wheel and the ground is always equal to the radius of the wheel. So, this paper determines the height of the vehicle by this distance.In practice, the scheme greatly improves production efficiency and improves the quality of animation products. But the plan is still to be improved, such as in the designated points, the vehicle speed and other aspects of the control.

\section{Acknowledgments}

This work is supported by the Natural Science Foundation of Shandong Province Grand No.ZR2016FM20.I am particularly grateful to it.

\section{References}

[1]Zhong Tie,Maya 2012 learning manual completely,first ed.,tsinghua university press,2013

[2] World ToolK it referen cem anua lRelease 9[ M] . Englineering An imation, Inc. 1999. 194 196.

[3]DonaldH earn, M Pau line B aker. computer graphics[ M] . Electronic Industry Publishing House, 1998.

[4] Sanhui Zhang. College Physics (first volume)[ M] . Tsinghua university press, $1999.250 \sim$ 253, $37 \sim 39$. 
[5]T. Chung, J. Kim, K. Yi. Human in the loop evaluation of a vehicle stability controller using a vehicle simulator. Automotive Technology, 2004.5:109.

[6] Vaibhav Govil, Stacy Lovell, Piriyakala Suresh, Qiong Wu. A Hing-Resolution Wide-Screen Display for Simulators and Virtual Reality. Proceedings of the Human Factors and Ergonomics Society 48th Annual Meeting.2004:2131 2133.

[7] A. Ikeda. Study of driver characteristics using driving simulation considerations of difference in accident avoidance performance due to age .JSAE Rev.23, 219(2002).

[8] Jong-Hae, Yoshimichi, Soichiro. Acquisition and Modeling of Driving Skills by Using Three Dimensional Driving Simulator. IEICE Trans Fundamentals. MARCH 2005 ( Vol. E88-A No.3) : 770 778.

[9] L.Dornand, D.Barker. The effects of driver training on simulated driving performance. Accident Analysis and Prevention, 2004.

[10] A. Ikeda. Study of driver characteristics using driving simulation considerations of difference in accident avoidance performance due to age .JSAE Rev.23, 219(2002). 